\title{
Reconstruction of extremity long bone defects with vascularized fibula bone grafts
}

\author{
Corinne Wee', Daniel Ruter ${ }^{2}$, Steven Schulz ${ }^{3}$, Geoffroy Sisk ${ }^{3}$, Julie West ${ }^{3}$, Scott Tintle ${ }^{4}$, lan Valerio ${ }^{3}$ \\ 'Division of Plastic and Reconstructive Surgery in the Department of Surgery, Case Western Reserve University, Cleveland, OH \\ 44106, USA. \\ ${ }^{2}$ Division of Plastic and Reconstructive Surgery in the Department of Surgery, Albany Medical College, Albany, NY 12208, USA. \\ ${ }^{3}$ Department of Plastic and Reconstructive Surgery, The Ohio State University, Columbus, OH 43212, USA. \\ ${ }^{4}$ Department of Orthopaedic Surgery, Walter Reed National Military Medical Center, Bethesda, MD 20889, USA.
}

Correspondence to: Prof. Ian Valerio, Department of Plastic and Reconstructive Surgery, The Ohio State University, 915 Olentangy River Road, Ste 2100, Columbus, OH 43212, USA. Email: ian.valerio@osumc.edu

How to cite this article: Wee C, Ruter D, Schulz S, Sisk G, West J, Tintle S, Valerio I. Reconstruction of extremity long bone defects with vascularized fibula bone grafts. Plast Aesthet Res 2019;6:12. http://dx.doi.org/10.20517/2347-9264.2019.02

Received: 6 Jan 2019 First Decision: 17 Apr 2019 Revised: 9 May 2019 Accepted: 30 May 2019 Published: 21 Jun 2019

Science Editor: Dr. Matthew L. lorio Copy Editor: Cai-Hong Wang Production Editor: Jing Yu

\begin{abstract}
Aim: Composite tissue defects encompassing bone and/or isolated bony defects can pose a surgical challenge; however, their reconstruction is critical for successful functional limb salvage. These cases become increasingly problematic as secondary defects, following multiple nonvascularized grafting attempts resulting in complex bony nonunion. Herein, our experience utilizing fibula vascularized bone grafts (VBGs) for bone restoration will be presented to demonstrate their utility in a variety of reconstructions for limb salvage.
\end{abstract}

Methods: This is a case series describing a series of vascularized fibula grafts for extremity reconstruction performed by a single academic surgeon over multiple institutions in seven years.

Results: Twenty-seven (27) total VBGs met inclusion criteria and underwent reconstruction for traumatic (16), oncologic (6) and chronic degenerative (5) etiologies. Bony union was achieved in 26 of 27 cases.

Conclusion: The decision-making process for bony reconstruction in these scenarios is difficult and multivariable. Fibula VBGs can provide a single-stage solution for autologous bony and soft tissue replacement of large or complex bone defects and can often be superior options compared with non-vascularized bone grafts or non-bone internal fixation techniques. Their osteogenic potential is unmatched by allogenic or synthetic substitutions. These benefits are evident in a variety of clinical settings such as pediatrics, oncology and trauma. 
Keywords: Vascularized bone, vascularized bone grafts, composite extremity defect restoration, bone reconstruction, free tissue transfer, microsurgery

\section{INTRODUCTION}

Segmental long bone defects and bony nonunions can arise after traumatic injury, oncologic resection, or osteomyelitis. Establishing a stable bony framework is critical to successful limb salvage; however, bony reconstruction often presents complex challenges to the reconstructive surgeon with seemingly limited available options. One must consider a variety of factors when selecting the appropriate treatment modality from a multitude of limb salvage options. Among these considerations are the surgeon's training background and experience, location and size of defect, associated injuries, availability of soft tissue coverage, and patient comorbidities.

Research and technology have led to a surge of products for bony reconstruction that obviate the need for autologous bone harvest, avoiding the potential donor site morbidity. These include both allografts and synthetic products such as bone morphogenic protein (Medtronic, Minneapolis, MN), polymethylmethacrylate (Zimmer Biomet, Warsaw, IN) and tricalcium phosphate (Depuy Synthes, New Brunswick, NJ). Many of these technologies possess osteoconductive and/or osteoinductive properties, or can be combined with another product to achieve both. Clinical studies suggest that both allograft and autograft can lead to adequate healing in a well-vascularized wound bed, with the end points being time to incorporation and lack of wound healing complications such as nonunion ${ }^{[1]}$.

However, these products are subject to their own set of limitations and disadvantages, including the risk of disease transmission, infection and autoimmune rejection. More importantly, the Diamond Model of fracture healing describes 4 requirements for adequate fracture healing which are best met by autologous reconstruction: osteogenic cell supply, an osteoconductive scaffold, growth factors, and a stabilized environment ${ }^{[2]}$. In particular, the lack of osteogenic cell supply in allograft and synthetic materials may be the reason they have demonstrated inferior outcomes in critically sized defects $>1 \mathrm{~cm}$, or in those of increasing severity ${ }^{[3]}$.

Thus, autogenous bony reconstruction remains the gold standard for bone loss. As there are many options to consider in this category, an initial size-based elimination approach can be helpful. Intramedullary nail, external fixation, and internal fixation techniques are options when there is no bone gap. When there is a bone gap, more complex procedures are appropriate depending on the size of the gap; these are further illustrated in Table 1.

While it serves as a good starting point, bone gap size is only one of many factors contributing to decisionmaking in orthopaedic and orthoplastic reconstructions. In the senior authors' practices, the utility and versatility of vascularized bone grafts (VBGs) for challenging bony reconstruction has expanded limb salvage options for many patients treated at our medical centers. The following cases demonstrate how fibula VBGs can optimize restoration of large segmental bone defects and resolution of nonunion cases to achieve definitive bony healing.

\section{MATERIALS/METHODS}

This is a retrospective case series of VBGs performed by a single surgeon over a seven-year period. Twentyseven (27) total VBGs met inclusion criteria and underwent reconstruction for traumatic (16), oncologic (6) and chronic degenerative (5) etiologies. Patient age ranged from 5 to 64 years with the majority of patients being younger than 30 years old. Anatomical bony reconstructions included 13 upper $v s .11$ lower extremity 


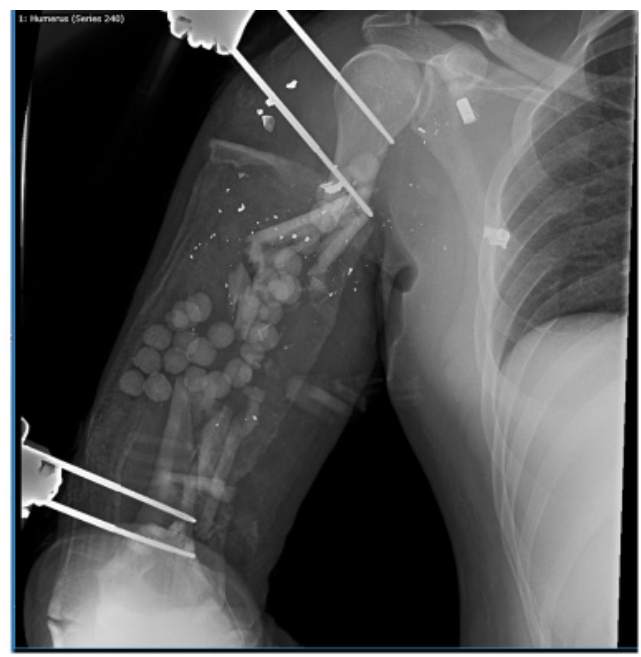

Figure 1. Radiograph showing severely comminuted humerus fracture secondary to gunshot wound

Table 1 Characteristics of techniques for long bone reconstruction

\begin{tabular}{|c|c|c|c|c|c|}
\hline \multicolumn{6}{|c|}{ Reconstructive Options for Segmental Bone Defects } \\
\hline Technique & $\begin{array}{c}\text { Suggested } \\
\text { Maximum Length }\end{array}$ & $\begin{array}{c}\text { Minimum \# of } \\
\text { operations }\end{array}$ & Soft tissue component & Strengths & Limitations \\
\hline $\begin{array}{l}\text { Corticocancellous } \\
\text { bone graft }\end{array}$ & $<6 \mathrm{~cm}$ & 1 & No & $\begin{array}{l}\text { Single operation, } \\
\text { quick recovery }\end{array}$ & $\begin{array}{l}\text { Small defects with adequate soft } \\
\text { tissue coverage }\end{array}$ \\
\hline Cortical bone graft & $4-9 \mathrm{~cm}$ & 1 & No & $\begin{array}{l}\text { Single operation, } \\
\text { medium size } \\
\text { defects }\end{array}$ & $\begin{array}{l}\text { Small to medium defects with } \\
\text { adequate soft tissue coverage, } \\
\text { resorption and fracture with } \\
\text { longer grafts }\end{array}$ \\
\hline Induced membrane & $1-25 \mathrm{~cm}$ & 2 & No & $\begin{array}{l}\text { Technically } \\
\text { simple operation }\end{array}$ & $\begin{array}{l}\text { Medium to large defects with } \\
\text { adequate soft tissue coverage. } \\
\text { Two stages. Time to weight } \\
\text { bearing 6-18 months. }\end{array}$ \\
\hline $\begin{array}{l}\text { Distraction } \\
\text { osteogenesis }\end{array}$ & $6-25+\mathrm{cm}$ & 2 & No & $\begin{array}{l}\text { Early partial } \\
\text { weight bearing }\end{array}$ & $\begin{array}{l}\text { One } \mathrm{mm} / \text { day, soft tissue restricts } \\
\text { distraction, joint contracture }\end{array}$ \\
\hline Free fibula & $6-30 \mathrm{~cm}$ & 1 & $\begin{array}{l}\text { Multiple soft tissue } \\
\text { options (skin, } \\
\text { muscle, and chimeric } \\
\text { configurations) }\end{array}$ & $\begin{array}{l}\text { Large soft tissue } \\
\text { component, can } \\
\text { shape the bone }\end{array}$ & $\begin{array}{l}\text { Fibula often injured in lower } \\
\text { extremity trauma, soft tissue } \\
\text { contiguous with bone, iatrogenic } \\
\text { injury to another extremity }\end{array}$ \\
\hline
\end{tabular}

defects and 3 pelvic defects. Successful union or bone healing was observed in 26 of 27 cases, with the following complications noted: 2 cases of delayed soft tissue wound healing and 1 case of complete resorption of a fibula bone flap requiring salvage with an expandable megaprosthetic and additional soft tissue flap coverage.

\section{RESULTS}

The following are examples of cases performed within the case series mentioned above. Free fibula grafts in extensive trauma:

Case 1. A 38-year-old male presented after high-velocity gunshot wound (GSW) to his right arm, resulting in a severely comminuted fracture of his humerus [Figure 1]. He had segmental bone loss of the humerus and complete segmental loss of his radial nerve. The only realistic treatment option in this case was the induced membrane technique or a free vascularized fibular graft. We proceeded with the fibular graft as it provided immediate stability and the ability to begin early gentle range of motion of the humerus [Figures $2 \mathrm{~A}$ and $\mathrm{B}$ ]. Anastomosis was performed to a muscular branch off of the brachial artery. This patient is now one year out 

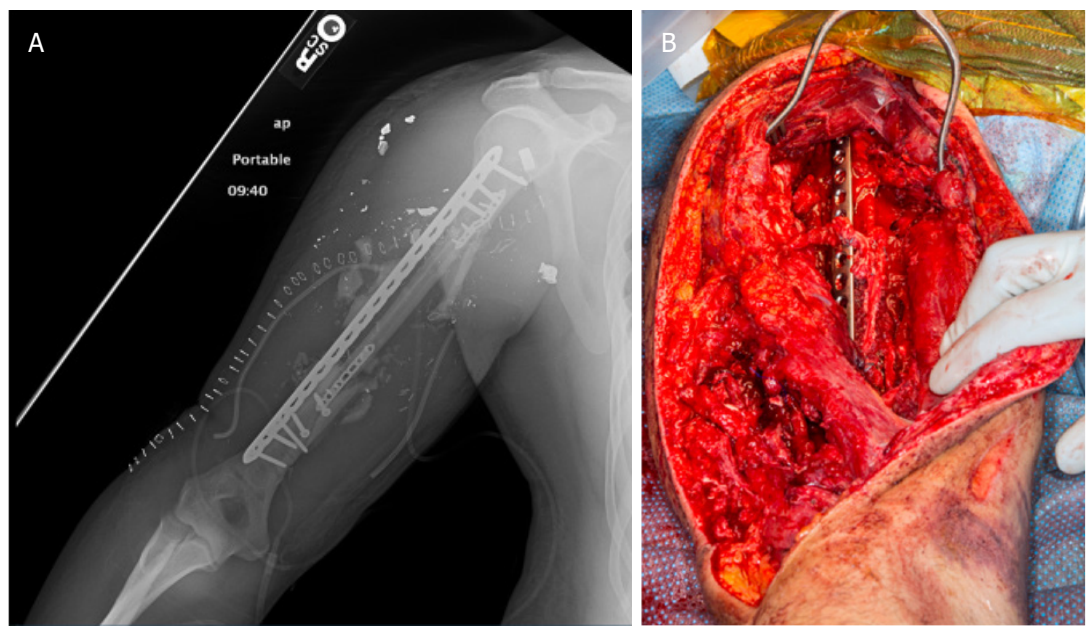

Figure 2. A: radiograph showing fixation of free fibula graft; B: Intraoperative photograph after fixation of free fibula graft

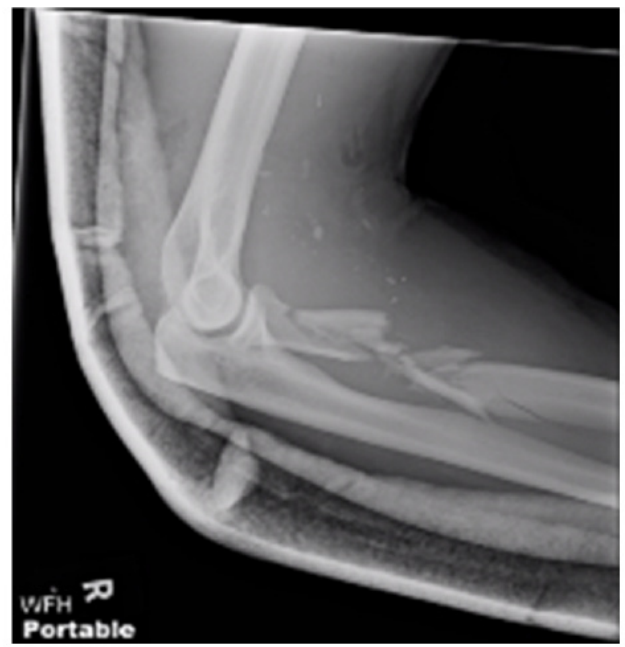

Figure 3. Complex radius fracture secondary to high-velocity gunshot wound

from his restorative surgery and has resumed an active lifestyle, including continuing his military service.

Case 2. A 36-year-old soldier presented with a complex radius fracture after suffering a high-velocity GSW to the proximal forearm [Figure 3 and 4]. The original plan was to fix the proximal radius with a bridging plate and place an antibiotic spacer. Intraoperatively, it was noted that the radial head and neck were not intact, and the longest radial head plate was not long enough to bridge the comminution. A free fibular graft was then utilized to bridge the $8 \mathrm{~cm}$ gap and provide immediate stability. The biceps tendon was excised from the bony fragment seen in the image and was attached to the fibula with suture anchors [Figure 4A-C]. The longest available radial head plate was utilized to secure the fibula in place to the proximal radial head. Note the intact posterior interossesous nerve draped over the fibula [Figure $4 \mathrm{D}$ ]. The patient had a radial nerve palsy prior to this surgery which resolved with time. He has since returned to full activity including pushups, pull-ups and weight lifting.

\section{Free fibula graft for oncologic reconstruction}

Case 3. We treated an 8-year-old male with a free fibula VBG following resection of a chondrosarcoma from his left humerus [Figure 5]. Free fibula VBGs are a good option for bone gaps greater than 6-7 cm, and have 

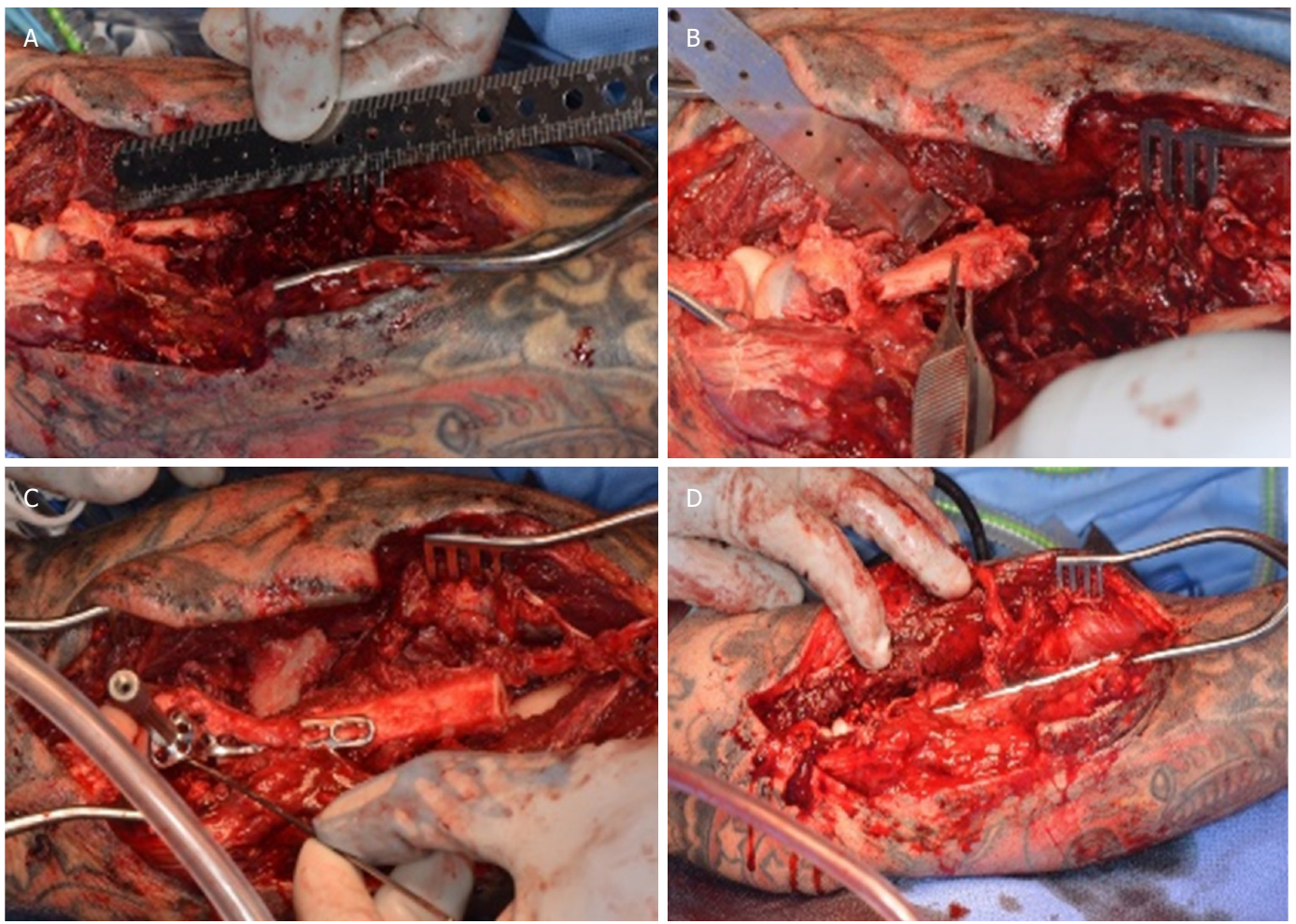

Figure 4. A: fracture separating radial head and neck with large bone gap; B: removal of bony fragment from biceps tendon; C: suture anchors used to attach biceps tendon to fibula graft; D: intact posterior interosseous nerve noted over fibula graft

been found in other reports to reliably achieve union at approximately 6 months ${ }^{[4]}$. A recent systematic review of free fibula flap reconstruction of humeral bone defects after oncologic resection found $93 \%$ union in an average of 5 months ${ }^{[5]}$. VBGs in the oncologic setting have the additional advantage of increased durability in the face of adjuvant chemotherapy and radiation ${ }^{[6]}$. While allografts were previously utilized in oncologic reconstruction, these reconstructions were associated with a high fracture and nonunion rate of over 15\%, with over $80 \%$ of grafts failing in the setting of infection, and approximately $50 \%$ failing in the setting of fracture ${ }^{[7-9]}$. In a series of 20 patients who underwent both upper and lower extremity reconstruction with allograft after tumor resection, $60 \%$ required removal of their allograft followed by replacement with allograft of endoprosthesis due to failure ${ }^{[10]}$. While fibula grafts are also prone to complications such as fracture, they possess higher healing potential without the need for a major reoperation in comparison to allograft. Houdek et al. ${ }^{[1]]}$ reports a success rate of $100 \%$ after VBG fracture, with some patients undergoing operative fixation and others responding to conservative management alone. For the pediatric population, fibula VBGs have another advantage: the fibular head can be included to allow for bone growth while also replacing the humeral head in the glenohumeral joint for reconstruction of the humeral head and diaphysis in pediatric tumor resections. While classically, a proximal and distal segment are preserved at the donor site to protect the common peroneal nerve and maintain ankle stability, Shuck et al.$^{[12]}$ did not report any peroneal nerve deficits or instability with walking after removing the fibular head. This patient is currently one year out from surgery and has resumed participation in competitive athletics without significant functional upper extremity limitations or impairment.

Case 4. A 56-year-old female presented with chondrosarcoma of the humerus [Figure 6], which after necessary resection resulted in a large bony defect. We reconstructed this extensive defect with a free fibula bone flap using the Capanna technique [Figure 7]. The Capanna technique combines methods of bony reconstruction, using a VBG in conjunction with allograft bone. Variations of the technique have been described with regard to the specific placement of the VBG with respect to the allograft: it can be placed completely within the 


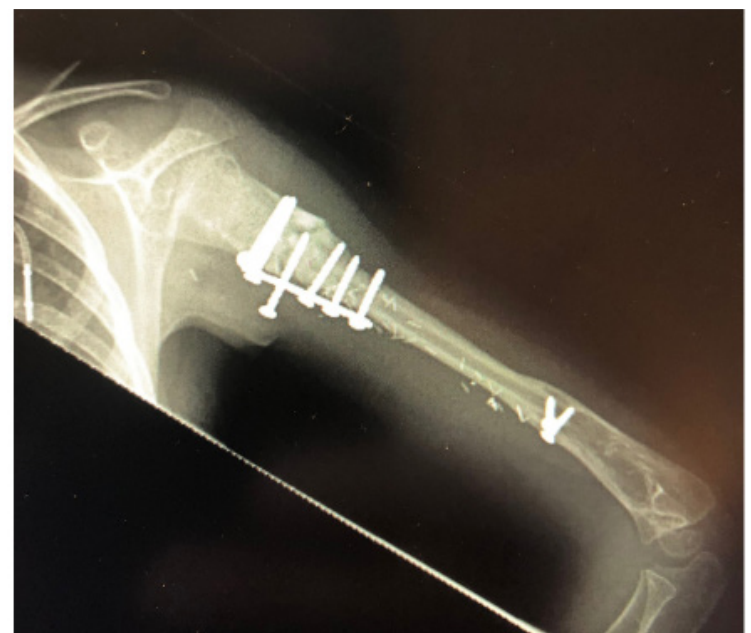

Figure 5. Fixation of free fibula flap to reconstruct bony defect after resection of a left humerus chondrosarcoma

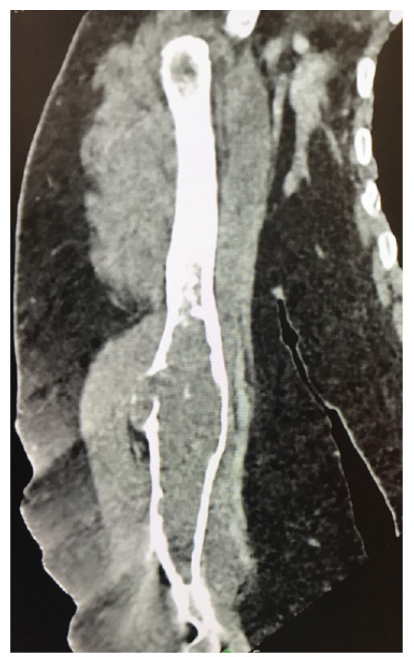

Figure 6. Radiograph showing extensive nature of humerus chondrosarcoma

allograft's medullary canal, partially within the canal, or alongside the allograft as an onlay ${ }^{[13-15]}$. Here, we chose to place the VBG partially within the medullary canal, inside a trough created through the bony cortex. VBGs are at risk for early fracture and thus require immobilization, sometimes for over a year depending on the anatomic location of reconstruction and rate of bony hypertrophy; the use of allograft contributes to early postoperative stability by bearing the load of bony fixation. In turn, VBGs provide osteogenic factors that allografts lack. This technique has been described in immediate and in delayed settings after resection with equivalent rates of union; this versatility allows for definitive reconstruction to be delayed to confirm surgical margins when they are in doubt ${ }^{[16]}$. While originally described for reconstruction after tumor resection, surgeons are beginning to use the Capanna technique in specific traumatic settings when risk for infection is low $^{[15]}$.

Case 5. A 63-year-old morbidly obese male with history of diabetes and chondrosarcoma of the femur presented with femur nonunion after he underwent neoadjuvant chemoradiation, tumor resection, and prior allograft placement complicated by infection and nonunion of the proximal allograft abutment [Figure 8A]. He required cane assistance in ambulation to reduce potential for hardware failure given his nonunion and body habitus. After a series of antibiotic nail exchanges, washouts, six months of negative microbacterial 


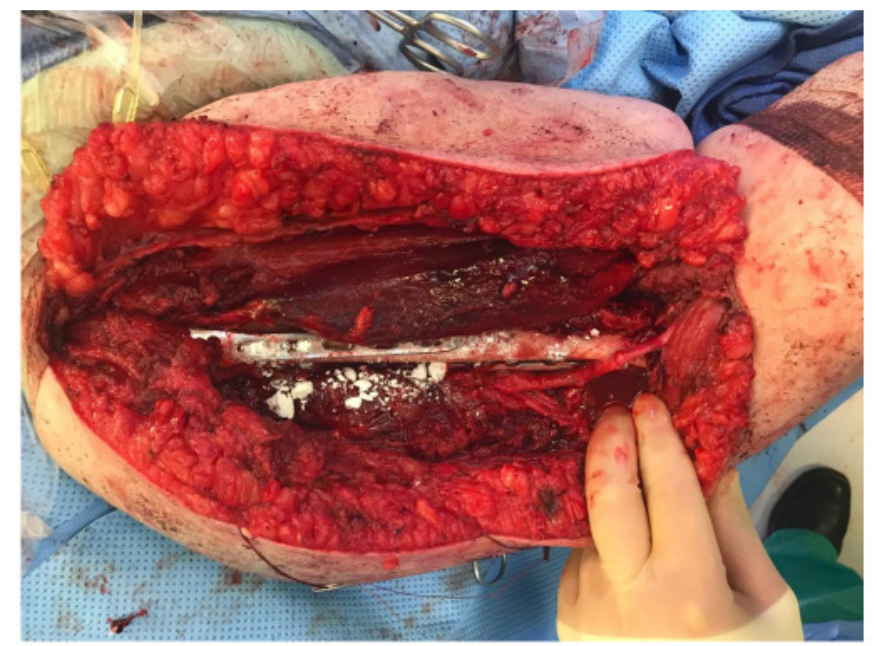

Figure 7. Free fibula used in conjunction with allograft, per the Capanna technique
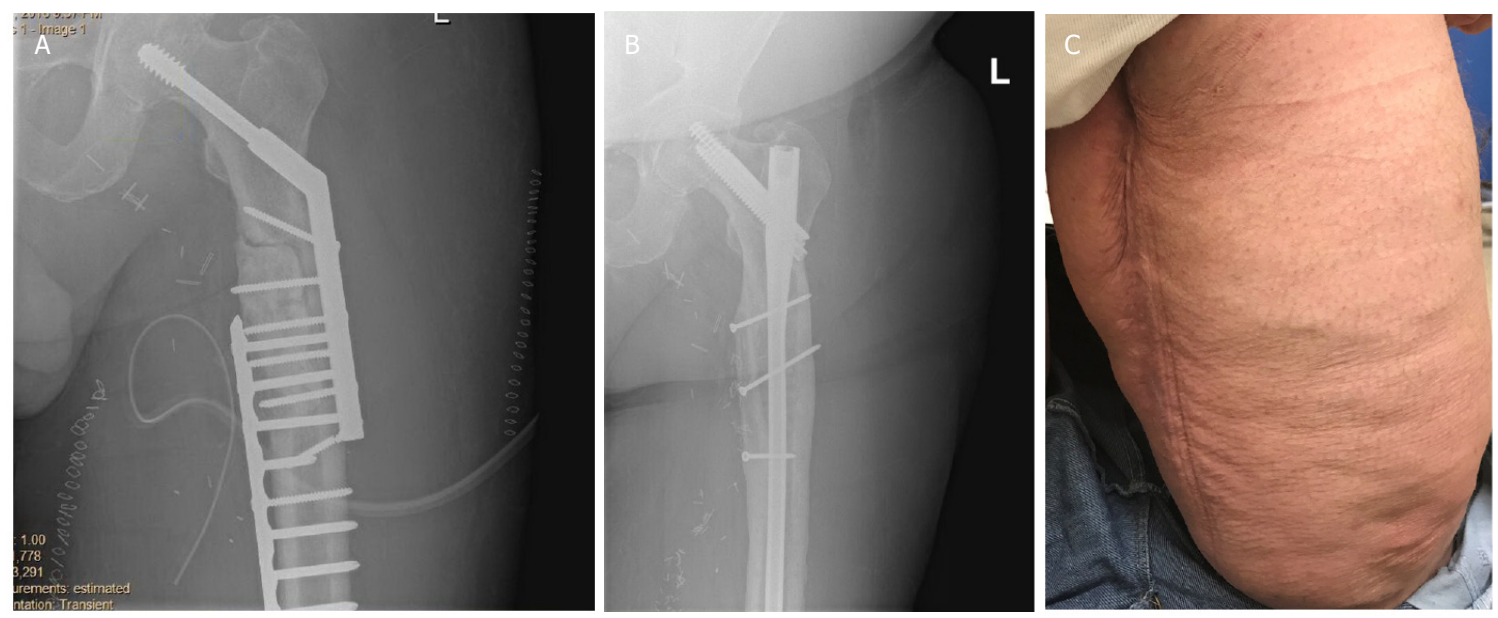

Figure 8. A: radiograph of case 5 before free fibula graft demonstrating nonunion; B: post operative radiograph of free fibula with IMN; C: two year follow-up with patient, who is ambulating and healing well

cultures and normalized limits of inflammatory parameters (White Blood Cell Count, ESR and CRP), the patient underwent reconstruction with a $14 \mathrm{~cm}$ free fibula VBG for his left femur nonunion. He is now oneyear post-reconstruction with radiographic and clinical evidence of complete bony healing, is ambulatory without cane assistance, and has returned to his full course of daily activities [Figures 8B, 8C].

\section{Free fibula graft for salvage of complex bony nonunion}

Cases 6 and 7. We treated two cases of middle-aged females who suffered traumatic tibial fractures complicated by nonunion despite failed allografting attempts [Figure 9]. Both of these tibial bone nonunions were definitively reconstructed with pedicled fibula VBGs [Figures 10A, 10B]. Pedicled ipsilateral fibula VBGs do not require advanced microsurgical techniques and can be especially helpful in patients who have failed previous bone grafting operations; they have also been reported in the reconstruction of oncologic tibial resections and in tibial plateau fractures requiring arthrodesis with acceptable surgical and functional outcomes ${ }^{[17-19]}$. This pedicle flap can be directly translocated as a "slide" or as a "turnover" technique i.e. rotated 180 degrees, and either technique can be based on antegrade or retrograde perfusion. Most commonly, the pedicle fibula flap is based on its antegrade flow pattern. Preoperative angiography or CT angiography can aid in assessing the peroneal as well as posterior and anterior tibial vascularity to ensure 


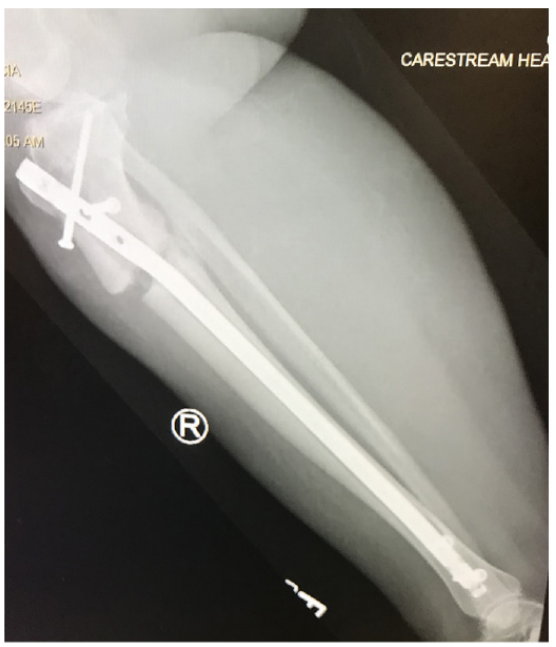

Figure 9. Radiograph demonstrating tibial nonunion despite previous fixation and allografting attempts
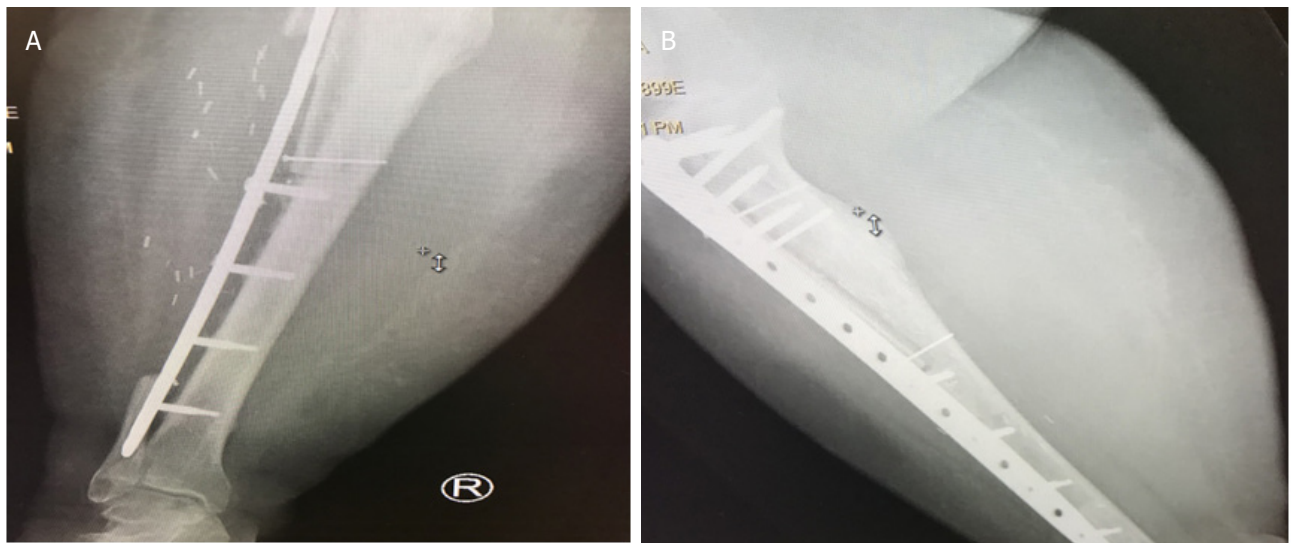

Figure 10. Radiographs demonstrating bony union following pedicled fibula reconstruction

viable blood flow to the ipsilateral fibula flap as well as preservation of dominant blood flow to the foot if the peroneal vessel is to be sacrificed distally. Of note, the pedicled fibula VBG can be difficult to harvest in traumatic or secondary salvage procedures due to extensive scarring, inflammation and abnormal anatomy.

\section{Free fibula graft as an osteocutaneous flap for composite reconstruction}

These cases represent additional advantages of the free fibula VBGs; when used as osteocutaneous flaps, they can reconstruct bony and associated soft tissue deficits in a single stage. With single-stage reconstruction, the patient is spared multiple flap reconstructions, avoids additional exposures to anesthetic risk, may preserve recipient vessels when performed in an end-to-side vascular anastomosis pattern, and eliminates the need to re-enter scarred wound beds for subsequent staged procedures ${ }^{[20]}$.

Case 9. This patient was 22-year-old army soldier who suffered a type I open both-bone forearm fracture complicated by infection that progressed to segmental infected nonunions [Figure 11]. The patient was treated with debridement, antibiotic spacer placement, and eventual free vascularized fibular graft to the ulna and a $3 \mathrm{~cm}$ non-vascularized segmental graft to the radius. The compromised soft tissue was replaced by the fibula skin paddle [Figure 12]. Both the radius and ulna healed successfully [Figure 13]. While his range of motion is decreased, the patient has returned to a productive life as a mechanic. 


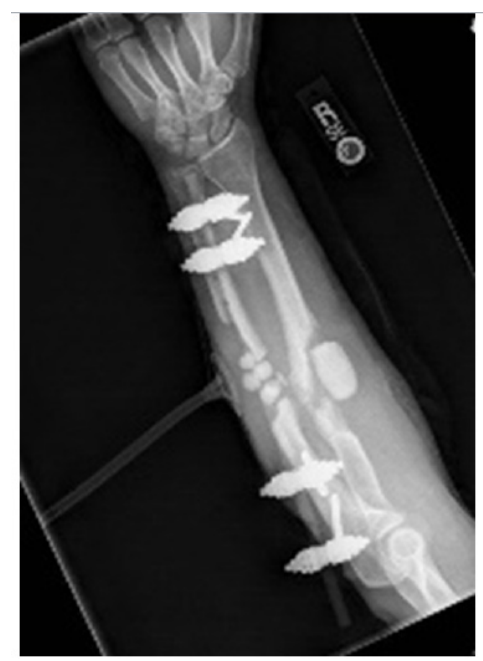

Figure 11. Radiograph demonstrating open both-bone forearm fracture

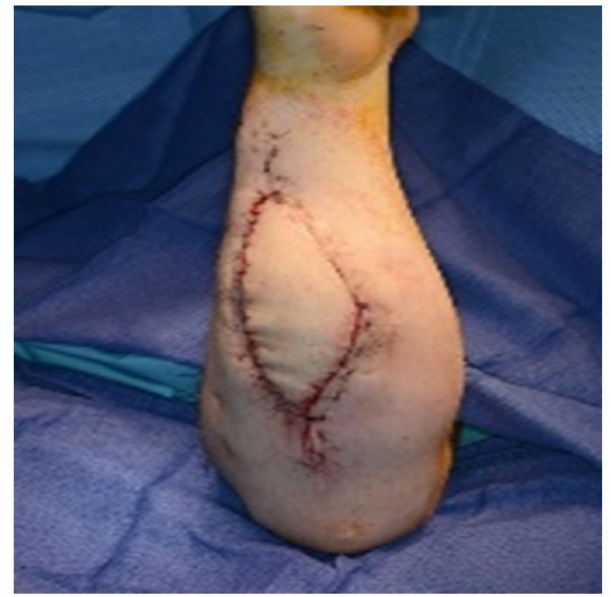

Figure 12. Osteocutaneous reconstruction with free fibula

Case 10. A 52-year-old male presented with hardware infection after his original distal tibia fracture was treated with plate fixation [Figure 14]. After necessary debridement, he was left with a segmental tibial and associated soft tissue defect. The ends of a free fibula graft were telescoped into the proximal and distal tibia and immediate stability was achieved. Small plates were utilized to ensure adequate fixation and a circular frame was then applied allowing for nearly immediate weight-bearing [Figure 15].

\section{DISCUSSION}

The decision-making process for reconstruction of segmental bone defects and osseous nonunion can be complex and multivariable. A multidisciplinary orthoplastic approach is recommended for optimal outcomes. Clear communication of reconstructive goals and options should be discussed among the orthoplastic surgery team. These goals should align with reconstructive goals, rehabilitation potential, and wound healing reserve of the patient at hand. In this illustrative case series, we sought to explore the utility and versatility of fibula vascularized bone grafts in reconstructing complicated bony defects and achieving bone union.

We found fibula VBGs to be an excellent method for single-stage bony reconstruction in patients with bony defects complicated by numerous factors, especially in cases with previously failed reconstruction. 


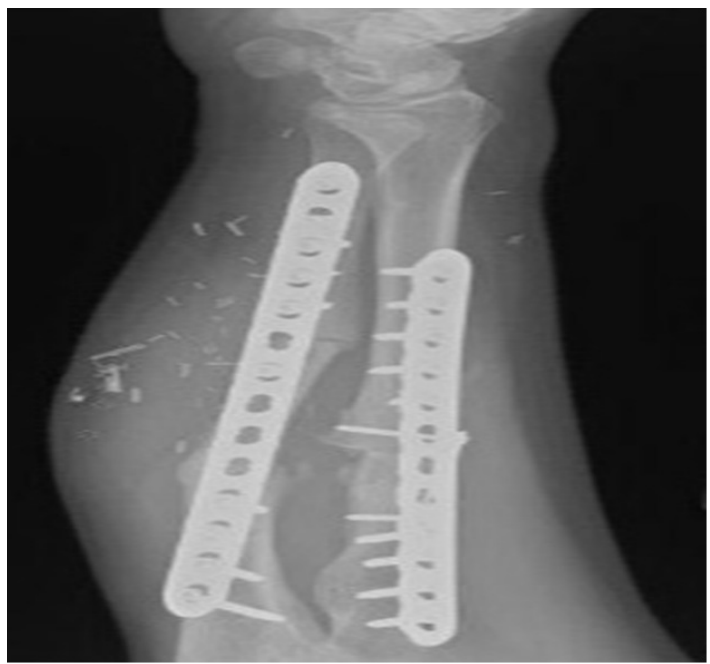

Figure 13. Radiograph after fixation with free fibula

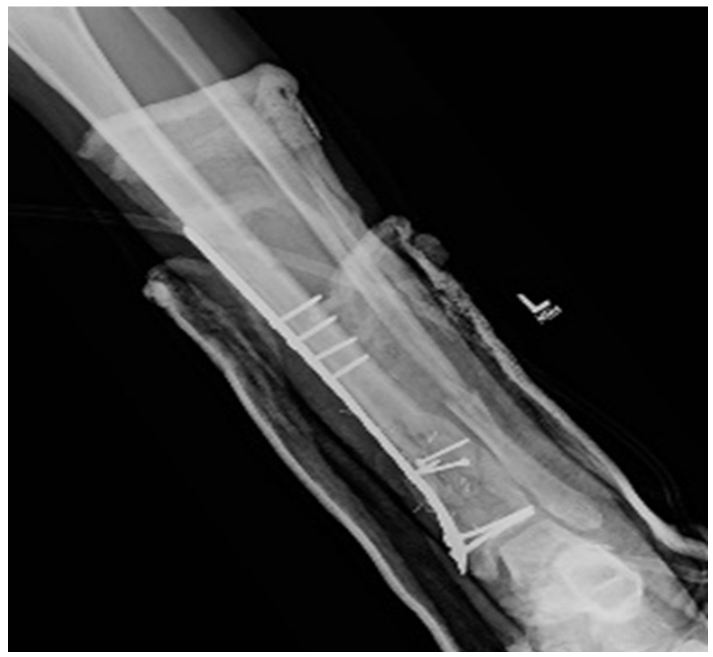

Figure 14. Previous distal tibia fixation complicated by hardware infection

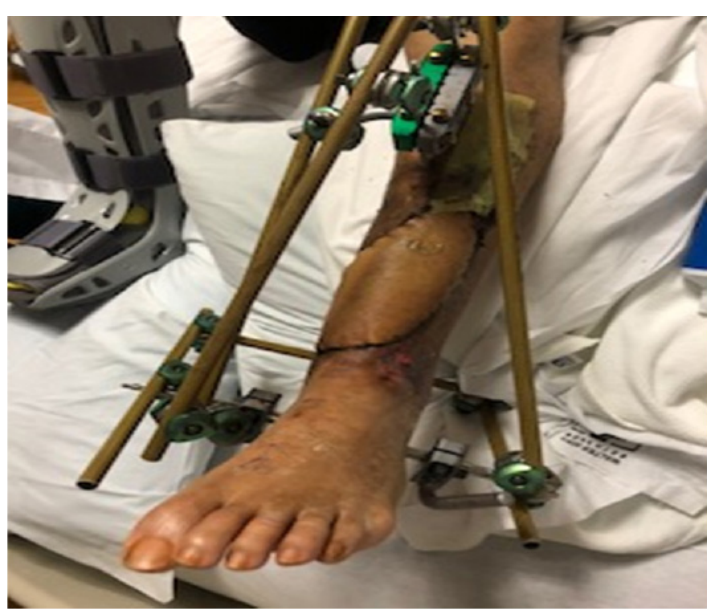

Figure 15. Osteocutaneous free fibula reconstruction with circular frame applied for improved immediate stability 
Allograft reconstruction can provide a shorter, less technically demanding reconstruction, but its success may be limited to well-vascularized wound beds of a smaller size. Current data suggests that larger defects with compromised vascularity may lead to a significantly higher rate of major complications in bony defects reconstructed with allograft when compared to autograft ${ }^{[2]}$. However, further study is required to explore the outcomes of different classes of allograft as the age and processing of the allograft may allow it to retain more osteoinductive properties. Autologous reconstruction can be performed in several ways and is also subject to its own limitations. The major reasons for the failure of traditional non-vascularized reconstructive techniques are large size of defect, residual nonviable bone secondary to avascularity or infection, and inadeqate soft tissue coverage ${ }^{[17]}$. In such challenging cases, fibula VBGs - in the form of bone flaps and osteocutaneous flaps - provide reconstructive options that incorporate stable vascularity and supply osteoinductive, osteoconductive and osteoprogenitor elements ${ }^{[20]}$. We found these properties of fibula VBGs to be useful in cases of severe trauma and composite tissue injuries, where the zone of injury often extends beyond what is perceived clinically or radiographically. In our oncologic and degenerative disease cohorts, fibula VBGs provide reliable blood flow to the bone as vascularity is often compromised in these situations due to chronic disease and/or radiation. Additionally, the use of the Capanna technique capitalizes on the ability of fibula VBGs to be used in combination with allografts to enhance vascular perfusion, allograft incorporation, and restoration of long bone osseous defects.

In conclusion, at our respective institutions, the orthoplastic surgeons have achieved excellent surgical outcomes, the most notable of which is high rates of successful bony union in patients with extremity bone defects and osseous nonunion cases from traumatic, oncologic, degenerative and congenital etiologies. The major disadvantages of fibula VBGs include longer operative times and higher technical demand, prolonged immobilization following surgery, and risk of early fracture. Fibula VBGs nonetheless provide an excellent reconstructive option for segmental bony defects and to address cases of failed nonvascularized nonunion grafting attempts in the extremities, and they offer promise in the efforts to improve outcomes and success in limb salvage. Our knowledge of the subject and our mastery of the techniques are continually expanding, fueled in part by multidisciplinary collaboration among trauma, oncologic, orthopaedic and plastic and reconstructive surgeons. It is our hope that this growing experience will lead to improved care for patients affected by limb-threatening bony pathology.

\section{DECLARATIONS}

\section{Acknowledgments}

The faculty and staff of the Departments of Plastic and Reconstructive Surgery and Orthopedic Surgery at the authors' institutions involved for supporting the orthoplastic surgery programs.

\section{Authors' contributions}

All authors made substantial contributions to conception and design of the study, performed data analysis and interpretation, as well as provided administrative, technical, and material support: Wee C, Ruter D, Schulz S, Sisk G, West J, Tintle S, Valerio I

\section{Availability of data and materials}

Not applicable.

\section{Financial support and sponsorship}

This work was supported by the IRB Protocol (2018E0888 and 2018E0350).

\section{Conflicts of interest}

All authors declared that there are no conflicts of interest. 


\section{Ethical approval and consent to participate}

Not applicable.

\section{Consent for publication}

Not applicable.

\section{Copyright}

(c) The Author(s) 2019.

\section{REFERENCES}

1. Dolan CM, Henning JA, Anderson JG, Bohay DR, Kornmesser MJ, et al. Randomized prospective study comparing tri-cortical iliac crest autograft to allograft in the lateral column lengthening component for operative correction of adult acquired flatfoot deformity. Foot Ankle Int 2007;28:8-12.

2. Giannoudis PV, Einhorn TA, Marsh D. Fracture healing: The diamond concept. Injury 2007;38:S3-6.

3. Cannada LK. A Randomized Controlled Trial Comparing rhBMP-2/ACS vs. Autograft for the Treatment of Tibia Fractures with Critical Size Defects. J Orthop Trauma 2019; doi:10.1097/BOT.0000000000001492

4. Adani R, Delcroix L, Tarallo L, Baccarani A, Innocenti M. Reconstruction of posttraumatic bone defects of the humerus with vascularized fibular graft. J Shoulder Elbow Surg;2008;17:578-84.

5. Landau MJ, Badash I, Yin C, Alluri RK, Patel KM. Free vascularized fibula grafting in the operative treatment of malignant bone tumors of the upper extremity:a systematic review of outcomes and complications. J Surg Oncol 2018;117:1432-9.

6. Canosa R, González del Pino J. Effect of methotrexate in the biology of free vascularized bone grafts. A comparative experimental study in the dog. Clin Orthop Relat Res 1994;291-301.

7. Berrey BH Jr, Lord CF, Gebhardt MC, Mankin HJ. Fractures of allografts. Frequency, treatment, and end-results. J Bone Joint Surg Am 1990;72:825-33.

8. Mankin HJ, Doppelt S, Tomford W. Clinical experience with allograft implantation. The first ten years. Clin Orthop Relat Res 1983;6986.

9. Mankin HJ, Gebhardt MC, Jennings LC, Springfield DS, Tomford WW. Long-term results of allograft replacement in the management of bone tumors. Clin Orthop Relat Res 1996;86-97.

10. Ogilvie CM, Crawford EA, Hosalkar HS, King JJ, Lackman RD. Long-term results for limb salvage with osteoarticular allograft reconstruction. Clin Orthop Relat Res 2009;467:2685-90.

11. Houdek MT, Wagner ER, Bishop AT, Shin AY, Rose PS, et al. Complications and long-term outcomes of free fibula reconstruction following resection of a malignant tumor in the extremities. Plast Reconstr Surg 2017;139:510e-9e.

12. Shuck J, Wood BC, Zarella C, Oh AK, Henshaw RM, et al. Near-complete humerus reconstruction in the pediatric patient with vascularized free fibula transfer. Plast Reconstr Surg Glob Open 2016;4:e1143.

13. Ridha H, Bernard J, Gateley D, Vesely MJ. Reconstruction of large traumatic segmental defects of the femur using segmental allograft with vascularized fibula inlay. J Reconstr Microsurg 2011;27:383-90.

14. Bakri K, Stans AA, Mardini S, Moran SL. Combined massive allograft and intramedullary vascularized fibula transfer: the capanna technique for lower-limb reconstruction. Semin Plast Surg 2008;22:234-41.

15. Venkatramani H, Sabapathy SR, Dheenadayalan J, Devendra A, Rajasekaran S. Reconstruction of post-traumatic long segment bone defects of the lower end of the femur by free vascularized fibula combined with allograft (modified Capanna's technique). Eur J Trauma Emerg Surg 2014;41:17-24.

16. Minami A, Kutsumi K, Takeda N, Kaneda K. Vascularized fibular graft for bone reconstruction of the extremities after tumor resection in limb-saving procedures. Microsurgery 1995;16:56-64.

17. Heller L, Phillips K, Levin LS. Pedicled osteocutaneous fibula flap for reconstruction in the lower extremity. Plast Reconstr Surg 2002;109:2037-42.

18. El-Sherbiny M. Long term behavior of pedicled vascularized fibular grafts in reconstruction of middle and distal tibia after resection of malignant bone tumors. J Egypt Natl Canc Inst 2008;20:187-95.

19. El-Negery A, Elmoghazy NA, Abd-Ellatif MS, Elgeidi A. Vascularized fibular medialization for reconstruction of the tibial defects following tumour excision. Int Orthop 2017;41:2179-87.

20. Yazar S, Lin C-H, Wei F-C. One-stage reconstruction of composite bone and soft-tissue defects in traumatic lower extremities. Plast Reconstr Surg 2004;114:1457-66.

21. Zhao Z, Yan T, Guo W, Yang R, Tang X, et al. Surgical options and reconstruction strategies for primary bone tumors of distal tibia: a systematic review of complications and functional outcome. J Bone Oncol 2019;14:100209. 